\title{
Effect of orthographic processes on letter position encoding
}

\author{
N. J. Pitchford and T. Ledgeway \\ University of Nottingham
}

J. Masterson*

University of Essex

\begin{abstract}
We investigate whether orthographic processes influence the identification and encoding of letter position within letter strings. To minimise word-specific effects, we adopt a visual letter search task that requires participants to identify a cued letter target among a random five-letter string. Using this paradigm, previous studies have shown that letter targets to the left are identified faster than those to the right of centre and letter targets in the initial, medial and final positions are identified faster than those in neighbouring positions. While the medial letter advantage is likely to arise from greater visual acuity at the point of fixation, the mechanisms responsible for the left-to-right, and exterior, letter advantage have yet to be determined. We show that: (i) search functions for most letters reflect the directional scanning process required for reading English orthography; (ii) search times are significantly faster for letter targets that appear in the most, compared with the least, frequent position in written words; and (iii) search times correlate significantly with positional letter frequency, especially in the initial and final positions. We propose that a combination of low-level visual, and higher-level orthographic, processes modulate the encoding of letter identities and position in written word recognition.
\end{abstract}

Before a written word can be identified it is commonly agreed that the constituent letters of the word must first be identified and their relative position within the word recognised. This process enables readers of alphabetic scripts to distinguish between words that comprise the same letters but arranged in a different relative order (e.g. 'made' and 'dame' or 'mate' and 'tame'). Past research has suggested that some letter positions are more influential than others for visual word recognition. In particular, the exterior letters of words appear to benefit from faster identification compared with letters forming the interior of written words. This advantage for the processing of exterior letters has been shown over a range of experimental paradigms involving the identification of written words and the processing of written text (e.g. Forster, 1976; Humphreys, Evett \& Quinlan, 1990; Humphreys, Evett, Quinlan \& Besner, 1987; Jordan, Thomas, Patching \&

*Prof. Jackie Masterson is now based at the Institute of Education, University of London, 25 Woburn Square, London, WC1H OAA, UK. 
Scott-Brown 2003a, b; McCusker, Gough \& Bias, 1981; Peressotti \& Grainger, 1995, 1999).

A processing advantage for exterior letters has also been shown on more low-level tasks that may appear to be only distally related to written word recognition, as they do not require explicit processing of written words. One such paradigm is the visual letter search task, in which participants are required to detect whether or not a previously cued target stimulus (e.g. T) is present within a random five-item string (e.g. XKTLP). Previous studies that have employed the letter search task show facilitation (i.e. greater accuracy and/or speeded responding) of the exterior letter positions (e.g. Hammond \& Green, 1982; Mason, 1975, 1982; Mason \& Katz, 1976). Thus, results from studies that have used the letter search task to investigate letter position encoding are consistent with other experimental paradigms that utilise real words and text reading. As such, it seems likely that performance on the letter search task reflects engagement with a system dedicated to the processing of written words.

The response time versus letter position function derived by skilled adult readers when searching for a target letter in a five-letter string is characterised by significant quartic and linear components that result in an M-shaped function that typically slopes upwards (e.g. Krueger, 1970). Target letters positioned to the far left of the array, in the initial position, are detected fastest, but the medial and final letters are also detected faster relative to their neighbouring letters occurring in the second and fourth positions. Similar tasks involving letter identification in horizontal strings have shown that when fixation centres on the medial letter (i.e. where visual acuity is greatest), identification accuracy drops with increasing eccentricity from the centre, except for the exterior letters, where accuracy in identifying targets is similar to that of the medial position (Averbach \& Corriell, 1961; Butler, 1975; Butler \& Merikle, 1973; Haber \& Standing, 1969; Merikle, Coltheart \& Lowe, 1971; Schwantes, 1978; Stevens \& Grainger, 2003; Wolford \& Hollingsworth, 1974).

In this paper, we explore the possible underlying mechanisms that give rise to the facilitation of exterior letters, especially the initial (most leftward) letter. The advantage for identifying letters at the ends of an array is unlikely to arise from reduced perceptual flanking ('crowding') as has previously been hypothesised (Bouma, 1970, 1973; Estes, 1972; Estes, Allmeyer \& Reder, 1976; Haber \& Standing, 1969; Van der Heijden, 1992) because reaction times for detecting non-letter shapes is slowest in these positions (Mason, 1982; Mason \& Katz, 1976; Hammond \& Green, 1982). When adults search for non-letter shapes response latencies for correctly detecting the presence of the cued target are a U-shaped (quadratic) function of the target position (order) within the array of items to be searched (Hammond \& Green, 1982). Non-letter shapes in the centre of the fiveitem array are detected faster than those appearing at the ends of the array, which is suggestive of a centre-out scanning process, in which there is a bias towards processing items in the fovea that decreases with eccentricity (Mason, 1982; Wagstaffe \& Pitchford, 2004; Wagstaffe, Pitchford \& Ledgeway, 2005).

As relative reaction times to identify letters and non-letter shapes are similar across positions 2, 3 and 4, but differ in positions 1 and 5, at the extremes of the array, this suggests that identification of letters and non-letter shapes located within the interior positions of the string may be mediated by the same low-level visual processes (relating to the location of the target relative to fixation). While a centre-out scanning process accounts for the slower identification of shape targets at the extremes of an array, different or additional mechanisms must operate to produce faster identification of letter 
targets at the ends of the array. Hammond and Green (1982) ruled out visual complexity as the sole explanation for the difference in response latencies for identifying letters and shapes at the extremes of a linear array, as shapes differing in complexity, both visual and semantic (i.e. nameability), consistently produced U-shaped search functions. Instead, top-down processes have been proposed to benefit exterior letter identification (Mason, 1982) although the nature or origin of these mechanisms has yet to be determined.

Some researchers have suggested that different attentional processes are used to scan arrays of letters compared with non-letter shapes. In contrast to non-letter shapes, which are thought to require a centre-out scanning process, letter identification has been argued to utilise a periphery-to-centre scanning process, in which the exterior letters of strings are processed before the interior letters (Butler, 1975; Butler \& Merikle, 1973; Bouma, 1973; Krumhansl \& Thomas, 1976; Merikle, 1974; Merikle \& Coltheart, 1972; Merikle et al., 1971; White, 1976), although it remains to be determined why this occurs. Furthermore, a periphery-to-centre scanning process per se also does not capture the other key feature of searching horizontal arrays of letters, that is the linear component, in which letter targets appearing to the left of the array are usually detected faster than those appearing to the right. Unlike non-letter shapes, the search function for letters typically slopes upwards, from left to right, as a function of target position. It is likely that this component reflects the serial left-to-right scanning process required for decoding English orthography, although the linear component is sometimes observed only when participants are asked to identify targets with their non-dominant hand (Green, Liow, Tng \& Zielinkski, 1996). As such, the linear component may arise from either a readingspecific process, such as serial directional scanning, or a task-related process, as when responding with the non-dominant hand, or a combination of these two processes. If the linear component arises from processes involved in written word recognition, this should be a principle characteristic of most letters, yet until now, individual search functions have not been reported for all letters of the English alphabet. Previous studies have either used a small set of nine consonants (Mason, 1982) or where a full set of letters has been used results have been collapsed across letter identity (Hammond \& Green, 1982).

In this study, we investigate whether top-down processes, originating from the operation of a specific system dedicated to the recognition of written words, influence performance on the letter search task. We explore two possible processes. First we examine whether the linear component of the letter search function reflects a directional scanning process consistent with reading habits. If so, we predict that the search functions of individual letters will show an advantage for processing letter targets appearing to the left, compared with the right, of the array, thus benefiting the initial letter position. As a linear component is sometimes observed only when participants respond with their nondominant hand (Green et al., 1996), in the present study we held hand of response constant. If, as we suggest, performance on the letter search task reflects the processing of orthographic input, and right-handed participants show elevated search times to targets appearing to the right, compared with the left, of the array, this would provide evidence to suggest that letter position encoding involves a serial scanning process in line with reading habits (e.g. Davis, 1999; Whitney, 2001). In contrast, if letter position encoding operates in parallel (e.g. Grainger \& Jacobs, 1996; Grainger \& van Heuven, 2003; McClelland \& Rumelhart, 1981), a linear component should not be a characterising feature of individual letters on this task.

Second, we explore the interesting possibility that the written word recognition system is sensitive to the effects of statistical learning, by which we mean the abstraction 
of the statistical regularities of the input orthography through prior exposure to print. Consequently the unique search function derived from visual letter search tasks may reflect the way in which orthographic input statistics have been encoded within the system dedicated to processing written words. Indeed, Vellutino, Scanlon and Tanzman (1994) consider orthographic processing as 'the ability to represent the unique array of letters that defines a printed word, as well as general aspects of the writing system such as sequential dependencies, structural redundancies, letter position frequencies, and so forth' (p. 314). Accordingly, performance on the letter search task may emerge as a product of these orthographic processes (Castles \& Nation, 2006).

Over the past decade there has been renewed interest in the possibility that the process of statistical learning may serve as a general mechanism underpinning different aspects of human cognition (see Saffran, 2002). Studies of spoken language acquisition have shown that human infants and adults are particularly adept at abstracting the invariant input statistics that are important for determining linguistic structure (such as word boundaries), while ignoring other irrelevant information in the acoustic signal (e.g. Saffran, 2001, 2002). Furthermore, the process of statistical learning does not appear to be constrained to human speech, but appears to be a general mechanism of perceptual learning that can be observed across different input domains, ranging from non-speech acoustic input (e.g. Saffran, 2001) to higher-order visual features (e.g. Aslin \& Fiser, 2004). Thus, if the process of statistical learning is a common mechanism of perceptual learning in general, its effects should be evident in visual word recognition, as the input orthography has statistical regularities that should be detected during the acquisition of reading skill.

One feature of an alphabetic orthography such as English that may be extracted through a process of statistical learning is the frequency with which letters appear in particular positions within words of a specified length (i.e. positional letter frequency), although the frequencies of larger sub-lexical units such as bigrams, onsets, rimes and syllables may also be extracted (e.g. McClelland \& Johnson, 1977; Nuerk, Rey, Graf \& Jacobs, 2000; Rey, Ziegler \& Jacobs, 2000; Vellutino et al., 1994). The frequency with which different letters of the English alphabet appear in written words differs both in terms of absolute frequency (i.e. how often a particular letter occurs in words per se) and positional frequency. Positional letter frequency refers to the number of times a particular letter appears in each of the positions within words of a specified length. For example, in five-letter words, the occurrence of the letter ' $A$ ' in positions 1 (e.g. ADULT), 2 (e.g. CAMEL), 3 (e.g. BLANK), 4 (e.g. PECAN) and 5 (e.g. EXTRA).

Grainger and Jacobs (1993) showed evidence of the effects of positional letter frequency operating in written word recognition in a series of primed lexical decision experiments with skilled readers of French. In these experiments, they manipulated the positional frequencies of the letter shared by the prime and the target string in relation to the overall positional letter frequency of the target. Results showed that the word-initial superiority effect found by other researchers (e.g. Humphreys et al., 1990) was eradicated when the stimuli were carefully controlled for positional letter frequency and neighbourhood effects. Furthermore, a recent study by Lupker, Perea and Davis (in press) showed that letter frequency modulated the degree of lexical priming for skilled readers of English. In a primed lexical decision task, transposed-letter primes (such as 'sibazle' for the target 'SIZABLE'), where the transposed letters are of relatively low frequency, were more effective than transposed-letter primes (such as 'heteric' for the target 'HERETIC'), where the transposed letters are of relatively high frequency. These studies 
suggest that skilled readers of alphabetic orthographies appear to abstract information pertaining to the frequency or likelihood with which certain letters appear in particular positions within written words from stored representations of real words within the orthographic lexicon.

If, as we suggest, sensitivity to the statistical regularities of orthographic input emerges as a product of learning to read, effects of positional letter frequency should also be observable in the performance of skilled readers on low-level tasks, that do not explicitly activate lexical representations, such as visual letter search, assuming, of course, that these tasks tap into the written word recognition system. As using random letter strings instead of real words in tasks investigating letter position encoding minimises the effects of top-down processes (such as neighbourhood effects, e.g. Grainger \& Jacobs, 1993) that may influence performance (Perea \& Pollatsek, 1998), any effect of positional letter frequency found on a visual letter search task would suggest that these effects operate at, or modulate, a relatively early stage of orthographic processing, such as abstract letter encoding.

In order to investigate whether performance on the visual search task is modulated by positional letter frequency, we presented each of the 25 letter targets in each of the five stimulus positions across the array on four occasions so as to produce a mean RT (ms) for each of the 25 letters. This enabled us to compare search times with correctly identified letter targets appearing in the most, relative to the least, frequent position within written five-letter words, and to correlate search times for each of the 25 letter targets with the frequency counts for the corresponding letters and positions derived from five-letter words. We used the counts generated by Mayzner and Tresselt (1965, see p. 14) that refer to the number of times a particular letter appears in each position of five-letter words, based on a sample of 20,000 words. For example, the frequency counts for the letter 'A' within five-letter words are 255,349, 383, 165 and 12 for positions 1, 2, 3, 4 and 5, respectively.

If positional letter frequency is an orthographic property that influences the identification of letters within letter strings, search times should be faster for identifying target letters appearing in the string position for which the letter target appears most frequently in five-letter words compared with the string position where the target letter is least frequent in written words. For example, for English five-letter words, the letter 'A' appears most frequently in position 3 (383) and is least frequent in position 5 (12). Thus, response times (RT) to identify the target letter 'A' within a random array of five letters should be faster when the target letter appears in the third (e.g. KLAMQ) compared with the last (e.g. HFRTA) position. In addition, if positional letter frequency influences the process of letter position encoding detection times should be inversely related to positional letter frequency. This relationship may be particularly prominent for exterior letters if these letter positions have a privileged status in written word recognition (i.e. letters with higher positional frequencies should be detected faster than letters with lower positional frequencies in the exterior positions).

\section{Method}

\section{Participants}

Twenty-eight individuals with normal or corrected-to-normal vision participated in the study. All were drawn from the undergraduate and postgraduate populations at the 
University of Essex and the University of Nottingham and were monolingual English speakers (age range 18-35 years). None of the participants presented with reading difficulties or were reported to have experienced difficulties with learning to read. All participants were right-handed and responded 'Yes' with their right hand and 'No' with their left hand. ${ }^{1}$

\section{Design, apparatus and stimuli}

A repeated-measures design was employed in which the two independent variables were position of the target letter within the string (5) and letter identity (25). Stimuli were presented on a CRT Mitsubishi monitor (resolution of $1,024 \times 768$ pixels, with a refresh rate of $85 \mathrm{~Hz}$ ) under the control of an Apple Macintosh G4 computer using custom software developed in our laboratory written in the $\mathrm{C}$ programming language. Stimuli consisted of 25 letters of the English alphabet, i.e. all of them with the exception of $\mathrm{O}$, which as Green et al. (1996) point out, could be confused with the digit zero. All stimuli were high contrast ( $\sim 95 \%$ Michelson contrast) and presented in white, upper case, Geneva, 36-point font against a uniform black background. At a viewing distance of $57 \mathrm{~cm}$ each letter subtended a visual angle of $\sim 1^{\circ} \times 1^{\circ}$ on average. There was no fixation point and participants were thus free to fixate on the screen.

A total of 1,000 experimental forced-choice trials were given. On each trial a letter cue appeared in the centre of the screen for a duration of $1,000 \mathrm{~ms}$. Following an intertarget-letter-string-interval of $500 \mathrm{~ms}$, where the screen went blank, a five-letter string was then presented in the centre of the screen (see Figure 1). For 50\% of the trials $(500$ trials) the cued letter appeared as the target (i.e. was present) in one of the five positions within the letter string and for the remaining 50\% of trials (500 trials) the letter cue was absent from the stimulus string. The order of these trials was randomised.

Each of the 25 letters was presented as a cue on 40 trials. For 20 of these trials the cued letter appeared as a target in either position 1,2,3,4 or 5 of the stimulus string (four

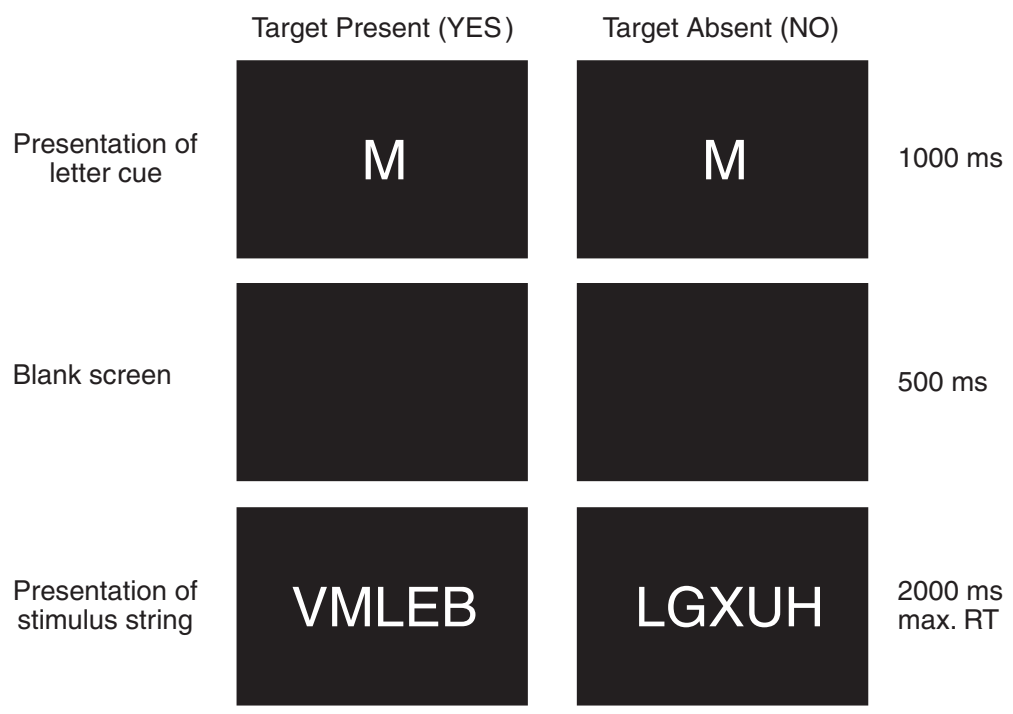

Figure 1. Schematic illustration of the letter stimuli used for the visual letter search task. 
trials/position). Thus the frequency with which each letter appeared as a cue was equated across the experimental trials in which the target was either present or absent.

The letter strings were composed of five different letters. In the case when the target letter was present the remaining four letters in the string were selected at random without replacement. For trials in which the target letter was absent all five letters in the string were chosen at random. There was a constraint on successive presentation of target letters, such that the same target letter could not be presented in the same position within the string on consecutive trials. However, there were no constraints on the composition of the letter strings, such that, purely by chance, letter strings could be non-pronounceable (e.g. FJLPX), pronounceable pseudowords (e.g. BLATE) or pronounceable real words (e.g. PLANK), although the probability of the latter two occurring was extremely low. From a sample of 1,000 randomly generated letter strings (the same number of stimuli presented to each participant in this experiment) none were real words, only $4.6 \%$ were pronounceable pseudowords and the remaining $95.4 \%$ were non-pronounceable letter strings. ${ }^{2}$

\section{Procedure}

Each participant was tested individually in a quiet laboratory. The experiment started with a standard set of instructions displayed on the screen. Participants were asked to press the/key on the computer keyboard with their right hand when they thought the target letter was present in the five-letter array and to press the $\mathrm{Z}$ key with their left hand when they thought the target letter was absent from the five-letter array. They were encouraged to respond as quickly, yet as accurately, as possible.

Following the instructions 10 practice trials were given to familiarise participants with the task, after which the 1,000 experimental trials were given. Each trial consisted of presentation of the letter cue, followed by a brief interval during which the screen was blank, and then the presentation of the five-letter string (see Figure 1). The letter string remained on the screen until the participant made his or her response by pressing one of the response keys. The following trial was then initiated after an inter-trial interval of $1,000 \mathrm{~ms}$. Auditory feedback was provided at the end of each trial for incorrect responses only by means of a brief auditory tone. After every 50 trials participants were asked, by a written message on the screen, if they wanted to pause for a few seconds. In addition, participants could pause the experimental trials voluntarily, as desired, by pressing the space bar. The computer recorded valid RT for both correct and incorrect responses in $\mathrm{ms}$. The maximum valid response time was $2,000 \mathrm{~ms}$ and any values exceeding this upper limit were excluded from formal data analysis.

\section{Data analysis}

The experimental design enables five data points to be plotted which correspond to each of the five letter positions in which the target can appear. The resulting letter search function can then be characterised by four simple shapes: a straight line (linear); a U-shaped (quadratic) function; an $\mathrm{S}$ or Z-shaped (cubic) function; or an $\mathrm{M}$ or W-shaped (quartic) function, as used by previous researchers (e.g. Green et al., 1996). Analysis of variance (ANOVA), combined with planned orthogonal contrasts, was used to determine the characteristics of the letter search function produced in the following analyses. 


\section{Results}

The mean percentage of errors (i.e. to target stimuli presented in the stimulus string but not recognised as such) was extremely low across all five positions (position $1=0.1 \%$, position $2=0.09 \%$, position $3=0.06 \%$, position $4=0.06 \%$, position $5=0.1 \%$ ). Consequently, no further analysis of errors was conducted. For each participant mean RT (ms) for each of the five letter positions for each of the 25 target letters were entered into a 5 (letter position) $\times 25$ (letter identity) repeated-measures ANOVA. This revealed a significant main effect of letter position, $F(4,108)=17.956, p<.0001$, a significant main effect of letter identity, $F(24,648)=12.18, p<.0001$ and a significant interaction between letter position and letter identity, $F(96,2,592)=1.837, p<.0001$.

To investigate further the nature of the main effect of letter position planned orthogonal contrasts were conducted. As shown in Figure 2, there was a significant linear component, $F(1,27)=18.901, p<.0001$ that accounted for $66 \%$ of the variance and a significant quartic or M-shaped component, $F(1,27)=59.252, p<.0001$ that accounted for $29 \%$ of the variance. In addition, the direction of the linear component was positive, $r=.813(N=5)$, ns, two-tailed but $p<.05$, 1-tailed as mean RT generally increased from letter position 1 through to 5 . Thus, when collapsed across letter identity, our data exhibit the characteristic upward-sloping M-shaped letter search function found in other studies (e.g. Hammond \& Green, 1982).

The significant main effect of letter identity suggests that, when collapsed across letter position, participants identified some letters faster than others. Participants were fastest at identifying the letter $\mathrm{S}$ (mean RT $=623 \mathrm{~ms}, S D=118 \mathrm{~ms}$ ), and were slowest at identifying the letter $\mathrm{N}$ (mean RT $=730 \mathrm{~ms}, S D=162 \mathrm{~ms}$ ). Interestingly, mean RT for correctly identifying target letters, collapsed across string position, correlated significantly with mean letter frequency derived from five-letter English words, $r=-0.34(N=25)$, ns, two-tailed but $p<.05$, one-tailed.

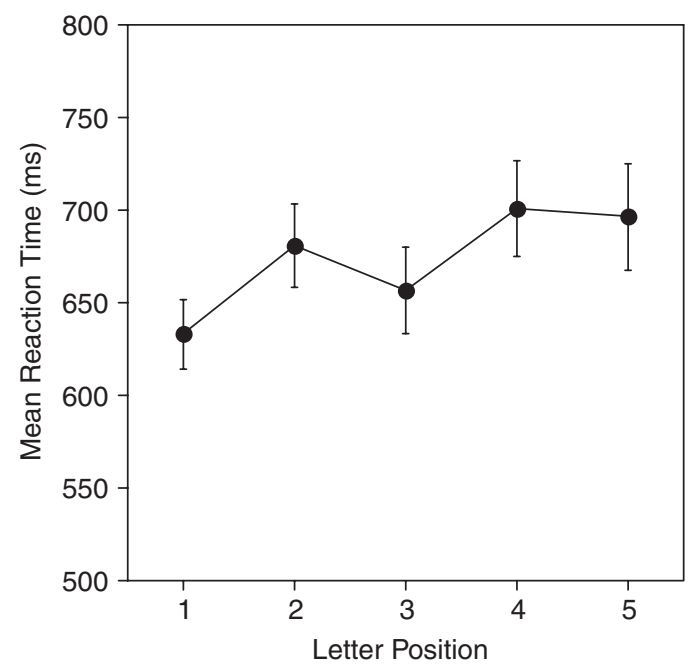

Figure 2. Characteristic upwards-sloping M-shaped letter search function produced by skilled adult readers of English. Error bars represent \pm 1 SEM. 
The significant interaction between letter position and letter identity suggests that the nature of the visual search function differs across letters. Letter search functions for each of the 25 target letters are plotted in Appendix A. To investigate the interaction, planned orthogonal contrasts were performed on the effect of letter position for each of the 25 letters. Appendix B reports the percentage of the total variance that was accounted for by each of the four different components (linear, quadratic, cubic and quartic) for each of the 25 letters. As can be seen from Appendices A and B, a linear component characterises the letter search function for most English letters, as the search function derived for 17 of the $25(68 \%)$ target letters of the English alphabet was captured mostly by a linear component. Furthermore, Appendix A shows there was a processing advantage for identifying a target letter located in the initial, most leftward position, compared with the second string position (at least), for 23 of the 25 (92\%) target letters, the only exceptions being the letters $\mathrm{N}$ and $\mathrm{V}$, where identification of these two letters was slower in the first, compared with the second, position of the stimulus string. In contrast, a processing advantage for identifying target letters appearing in the final string position was less consistent across the letters, as mean RT was lower in the fifth compared with the fourth string position for only 14 of the 25 (56\%) target letters (see Appendix A).

\section{Positional frequency analyses}

The significant inverse relationship between mean RT and mean letter frequency, collapsed across target position, reported above, suggests that participants exhibited a tendency to identify letter targets that appear more frequently in written words significantly faster than target letters that appear less frequently in English words composed of five letters. To investigate how search times may be related to the frequency with which letters appear in particular positions within five-letter words (i.e. positional letter frequency), two further analyses were performed.

First, we predicted that if positional letter frequency is an orthographic property that is extracted from stored representations of written words within the lexicon, participants should be faster at identifying letter targets that appear in the most, compared with the least, frequent position of written words. To investigate this hypothesis, we compared the mean RT for correctly detecting each of the 25 target letters in their most frequent position compared with the position in which they least frequently occur in five-letter English words. The mean frequency of the 25 target letters in their most common position was $270.48(S D=192.99$, range $=10-680)$ whereas the mean frequency for the 25 letter targets in their least frequent position in written words was 29.28 ( $S D=42.13$, range $=0-143$ ). According to the letter frequency counts of Mayzner and Tresselt (1965), 4 of the 25 target letters have a positional letter frequency of zero in more than one position (these being the letters $\mathrm{J}$ in positions 2, 4 and 5; $\mathrm{Q}$ in position 3, 4 and 5; $\mathrm{X}$ in positions 1, 4 and 5; and Z in positions 1, 2 and 5). Thus, for these four letters, a mean RT was calculated from the string positions where positional letter frequency was zero. Consistent with our hypothesis, a paired $t$-test showed that participants were significantly faster at identifying letter targets that appear in the most (mean RT $=665 \mathrm{~ms}, S D=$ $128 \mathrm{~ms}$, range 465-1,028 ms) compared with the least (mean RT $=686 \mathrm{~ms}, S D=126 \mathrm{~ms}$, range 488-986 ms) frequent position in English five-letter words, $t(24)=-5.16$, $p<.0001$, two-tailed.

Second, we predicted that if performance on the letter search task is mediated by positional letter frequency, search times should correlate negatively with positional letter 


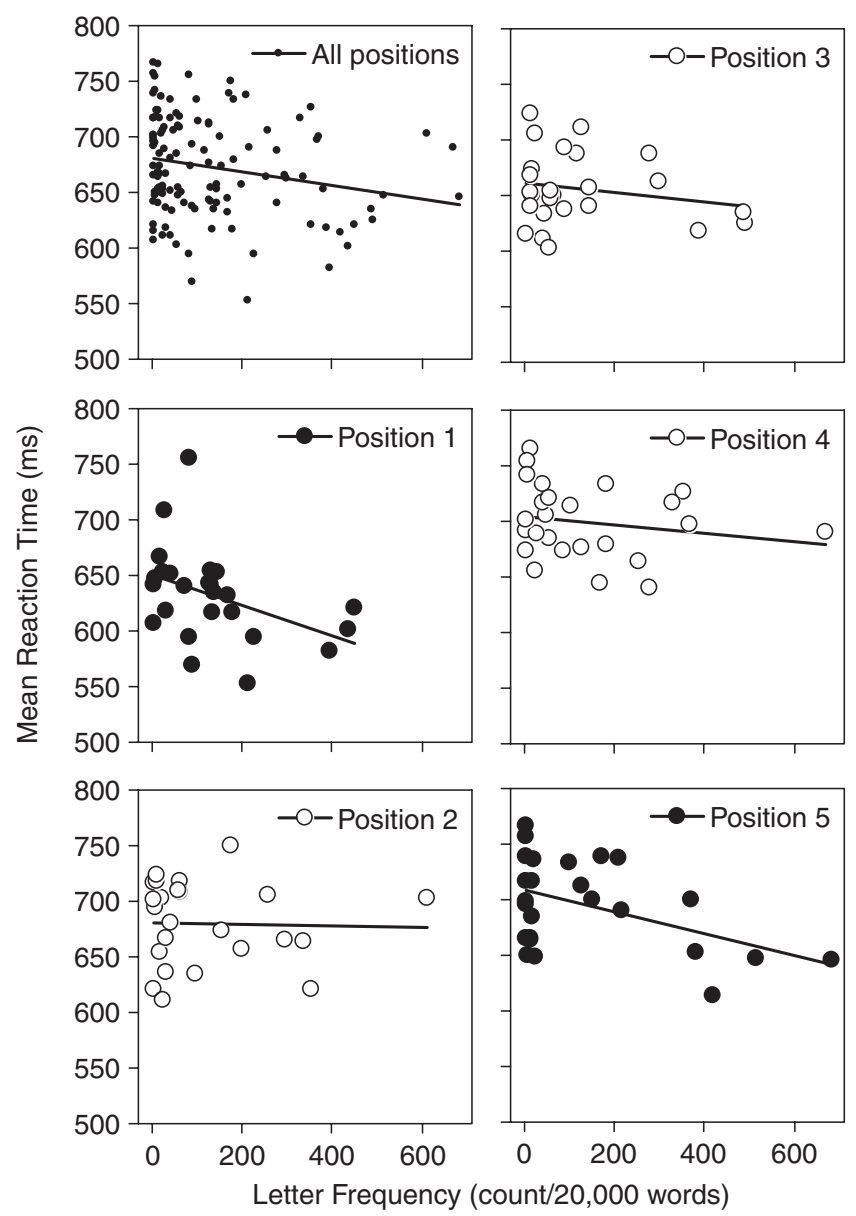

Figure 3. Scatterplots illustrating the relationship between mean reaction time and letter frequency plotted separately for each of the five positions within the stimulus string, and for all positions combined (top left). Filled circles illustrate correlations that are statistically significant (i.e. positions 1,5 and all positions).

frequency derived from written English words of five letters. Results, shown in Figure 3, revealed a moderate, yet significant, negative correlation between mean RT and positional frequency for each of the 25 letters in each of the five letter positions, $r=$ $-0.213(N=125), p=.002$, two-tailed. Although the correlation was in the predicted direction, the size of the correlation suggests a considerable amount of variation. To investigate if the source of this variation could be accounted for by particular letter positions, mean RT and letter frequency were correlated for each of the 25 letters in positions 1, 2, 3, 4 and 5. Interestingly, significant negative correlations were found only for letters appearing in position $1, r=-0.412(N=25), p=.04$, two-tailed and position $5, r=-0.459(N=25), p=.02$, two-tailed. In contrast, the correlation between RT and positional letter frequency was not significant for letter positions 2,3 and 4 (position 2, $r=-0.027, p=.898$; position 3, $r=-0.192, p=.364$; position 4, $r=-0.192, p=.364 ;$ all $[N=25]$, two-tailed). 


\section{Discussion}

This experiment investigated whether letter position encoding is mediated by top-down influences specific to the processing of written words: (i) a serial left-to-right scanning process utilised by English orthography; and (ii) an orthographic process related to lexical access. In particular, we explored whether facilitation of exterior letters found in letter search tasks and other more direct tasks of written word recognition may reflect sensitivities to statistical properties of the input orthography, such as positional letter frequency.

In keeping with previous studies, when collapsed across letter identity, our data show that skilled readers of English produce an upward-sloping M-shaped function, characterised by significant linear and quartic components, when searching for a prespecified target in a random five-letter string (Hammond \& Green, 1982; Mason, 1982). Thus, the two components that have previously been shown to differentiate letter search from non-letter search functions are also reflected in our data, as both relative facilitation of exterior letter positions and faster reaction times to targets appearing on the left compared with the right of the array were shown by our group of skilled adult readers, even though hand of response was controlled for.

We make the assumption throughout this paper that the unique M-shaped function derived by skilled readers of English when searching for letter targets embedded within random letter strings reflects processes that are typically engaged with orthographic input. This is because a different U-shaped function is generated when searching for non-letter shapes on the same task (e.g. Hammond \& Green, 1982; Mason, 1982). Thus, task demands alone cannot be responsible for facilitation of exterior letters on this task, as identification of non-letter shapes is slowest in these positions. Furthermore, the advantage for detecting letter targets appearing to the left compared with the right of the array cannot arise from processes employed only when searching horizontal arrays, as there is no evidence of such a directional scanning process operating when searching arrays of non-letter shapes (e.g. Hammond \& Green, 1982; Mason, 1982; Wagstaffe \& Pitchford, 2004; Wagstaffe et al., 2005). Rather, we propose that the linear component exhibited by skilled readers of English on the visual search task reflects a directional scanning process that is utilised for processing English orthography. However, it is possible that performance on the letter search task activates serial processes that do not occur in typical written word recognition, or that are more exaggerated. Even though performance when searching for non-letter shapes on the same task shows a different characteristic pattern this does not rule out the possibility that participants could be engaging in strategic search processes specific to letters during this task that are not typically engaged when processing written words.

In support of our supposition, a linear component was shown to characterise the search functions of most of the 25 letters used in our study. To investigate whether letter position encoding is influenced by a top-down directional scanning process consistent with reading habits, we reported, for the first time, individual search functions for all English letters (except for the letter $\mathrm{O}$ ) and determined their characterising components (see Appendices A and B). We argued that if letter position encoding is influenced by topdown processes related to reading habits (Davis, 1999; Whitney, 2001) this should be reflected in the search functions for individual letters. However, if letter position encoding operates in parallel (Grainger \& Jacobs, 1996; Grainger \& van Heuven, 2003; McClelland \& Rumelhart, 1981) a linear component would not be expected to 
characterise individual letter search functions, and may only arise when participants detect targets present in the stimulus string with their non-dominant hand (Green et al., 1996).

Our data showed that a linear component best characterised the search functions of 17 of the $25(68 \%)$ letters tested, and a processing advantage (i.e. faster detection time) was shown for the first, compared with the second, letter for 23 of the $25(92 \%)$ letters used in our experiment. This finding is important as it suggests that participants adopted a left-toright scanning process when searching for most letter targets located across the five string positions, which benefited identification of the initial (most leftward) letter, relative to other string positions. We believe this result is most likely to reflect a directional scanning process consistent with encoding English orthography rather than any taskrelated artefacts, as we kept hand of response constant in our study (see Green et al., 1996). This finding is thus more consistent with models of letter position encoding that incorporate a serial process (Davis, 1999; Whitney, 2001) than models that operate in parallel (Grainger \& Jacobs, 1996; Grainger \& van Heuven, 2003; McClelland \& Rumelhart, 1981).

Our results clearly show that when performing a letter search task skilled readers of English employ a directional scanning process, but to what extent does this depend on prior exposure to a particular orthography? A recent study by Ktori and Pitchford (in press) has a bearing on this issue. They showed that the transparency of an orthography influences the extent to which the left-to-right directional scanning process is exhibited on the letter search task. They compared the performance of skilled monoscriptal readers of English (a deep orthography) with that of skilled monoscriptal readers of Greek (a highly transparent orthography). The results showed that when searching for letters of their native orthography, the letter search function exhibited by skilled readers of Greek was significantly more linear than that exhibited by skilled readers of English. This finding is commensurate with the differences in transparency between the two orthographies, and suggests that the processes used for identifying letters and encoding their positions within letter strings are adaptive to the nature of the script to which the reader is exposed during reading acquisition. Performance on the letter search task appears to be sensitive to this.

Our data are also consistent with a recent study of letter identification in horizontal random consonant arrays conducted by Tydgat and Grainger (under review) using the Reicher-Wheeler task. They found that serial position effects benefited identification of targets located in the initial position for letters and digits but not for other non-letter shapes. They ruled out an explanation for these results based on the employment of different attentional processes for searching arrays of letters (periphery-to-centre) compared with non-letter shapes (centre-out) that has been proposed by previous researchers (Bouma, 1973; Butler, 1975; Butler \& Merikle, 1973; Krumhansl \& Thomas, 1976; Merikle, 1974; Merikle \& Coltheart, 1972; Merikle et al., 1971; White, 1976). This is because an advantage for letters and digits (but not shapes) appearing in the initial string position remained even when the presentation of letter, digit and shape trials was randomised (experiment 2) and when target letters were embedded within stimulus strings composed of non-letter shapes (experiment 3). Rather, Tydgat and Grainger (under review) tentatively put forward an account based on the assumption of adaptive receptive fields for letters and digits that are smaller in size, and are asymmetric in shape, compared with the receptive fields employed for processing arrays of non-letter shapes. This could enable serial position effects to result from a parallel encoding process, although further research is needed to test this hypothesis empirically. 
Consistent with our data, Tydgat and Grainger (under review) showed greater accuracy in identifying target letters located in the fifth string position, for blocked presentation of letter stimuli (experiment 1), although final letter facilitation was less pronounced than that found for the initial letter, and it disappeared when the type of stimulus was randomised across trials (experiment 2). In the current study, we found that participants identified letter targets appearing in the fifth, compared with the fourth, string position faster for 14 of the $25(56 \%)$ letters tested. This suggests that, compared with the initial letter, which appears to elicit a systematic processing advantage, facilitation of the final letter is a much more labile phenomenon. This may reflect a system of modified receptive fields, as speculated by Tydgat and Grainger (under review), or it may arise from a combination of the two top-down higher-level processes investigated in the present study. Systematic facilitation of the initial letter and more variable facilitation of the final letter may arise because only the initial letter position would benefit from both a serial scanning process and an orthographic process that is modulated by input statistics, such as positional letter frequency.

Furthermore, the present study found evidence to suggest that letter identification was speeded for positions in which letter targets appeared most often in written words, and this could not be readily accounted for by modification of low-level receptive fields as considered by Tydgat and Grainger (under review). Our data showed that participants were significantly faster at identifying target letters in random five-letter strings that appear in the most, compared with the least, frequent position derived from English words of five letters in length. ${ }^{3}$ In addition, significant negative correlations were found between response time to detect a target letter and positional letter frequency in the initial and final positions of the array. This finding is consistent with previous studies that have reported effects of positional letter frequency in tasks of written word recognition that require lexical access (Grainger \& Jacobs, 1993; Lupker et al., in press). As similar effects of positional letter frequency are found on the visual letter search task, which does not explicitly require accessing lexical representations, our results suggests that these effects must operate at, or modulate, a relatively early stage of processing orthographic input.

Based on these results, we suggest that positional letter frequency is a pervasive feature of written English, which skilled readers can utilise when processing written words, although larger sub-lexical units, such as bigram frequency, may also exert an influence (e.g. McClelland \& Johnson, 1977; Nuerk et al., 2000; Rey et al., 2000). These results are important as they imply that the processing advantage for exterior letters observed in studies of written word recognition may be determined, in part, by positional letter frequency. Furthermore, these findings should constrain and inform theories of how letter identity and letter position information are encoded within the reading system.

Sensitivity to statistical orthographic regularities would emerge implicitly within models of written word recognition (e.g. the interactive activation model of McClelland \& Rumelhart, 1981) that have feed-forward and feed-backward connections between multiple levels of representation, at the feature, letter and word level. Because in English, certain letters occur more frequently in certain positions in words (i.e. positional letter frequency is not uniform, Mayzner \& Tresselt, 1965), this heterogeneity will also be present at the word level. For example, within the orthographic lexicon of skilled readers there will be more five-letter word units that have a letter ' $A$ ' appearing in the first position than at the end. If top-down processing at the word level can modify resting activation states at the letter level, where relative position is encoded, this would provide 
a possible mechanism by which orthographic regularities present in the lexicon could influence letter identification.

Thus, even though the letter search task does not require explicit use of lexical processes, performance on this task appears to reflect engagement with a system dedicated to the processing of letter strings that is typically used in written word recognition. Initial presentation of the letter cue (e.g. T) is likely to activate feature and letter units, which, via feed-forward connections, will activate representations at the word level. If, as a result of orthographic learning, statistical regularities, such as positional letter frequencies, are extracted from the input orthography (Vellutino et al., 1994), time taken to detect the previously cued target when presented within the random five-letter string (e.g. XKTLP) may emerge as a product of orthographic learning (Castles \& Nation, 2006). Feed-backward connections from the word level could then modulate activation at the letter level. Thus, differential target detection time across the different string positions is likely to reflect orthographic regularities encoded within the system dedicated to the processing of written words.

Interestingly, the unique M-shaped letter search function also emerges when skilled readers search for a cued target letter (e.g. T) located within real five-letter words (e.g. EMPTY) (Wagstaffe, 2006). As written word recognition in skilled readers is considered to be automatic and unconscious, and not subject to strategic control (Ehri, 2005), presentation of a real five-letter word should result in automatic word recognition. The advantage that is shown for detecting exterior letter targets over neighbouring letter targets in both five-letter real words and random letter strings suggests the same orthographic processes are responsible.

The sensitivity to positional letter frequency shown in our data for letter targets appearing in the initial and final positions of a string suggests that the ends of words may be particularly important for speeded lexical retrieval in English. For example, fast processing of exterior letters would serve to rapidly constrain the set of candidate lexical entries corresponding to a written word, as only particular medial letters are plausible within English words of a specified length with particular exterior letter pairings. Furthermore, rapid processing of exterior letters would provide information about other factors that may speed word recognition, such as morphology, which is typically coded at the beginning (prefixes) and end (suffixes) of words.

It is important to point out that the significant correlations found between response time to detect letter targets and positional letter frequency in the exterior positions appear to be genuine orthographic effects and are not the result of differences in the range of RT or frequency values across the different positions. Both the range of target detection times (204, 138, 120, 124 and $153 \mathrm{~ms}$ for positions 1-5, respectively) and letter frequencies (445, 608, 487, 664 and 608 for position 1-5, respectively) were similar across the five positions of the string (as shown in Figure 3).

Furthermore, it is highly likely that the sensitivity to positional letter frequencies in the exterior positions arises at a level of abstract letter units as significant correlations were found for the exterior positions even though the frequency counts of Mayzner and Tresselt (1965) that were used were generated predominantly (although not exclusively) from lower-case letters and the letter stimuli we used were all upper case. Jones and Mewhort (2004) recently reported the correlation between the frequencies of upper and lower-case letters in written words to be 0.6337 , indicating some degree of case-sensitive variability. Although the letter counts published by these authors refer specifically to the occurrence of upper-case letters in samples of written text their counts are collapsed 
across letter position and word length; thus they were unsuitable for our analyses. Nevertheless if, as we hypothesise, information pertaining to the frequency with which letters appear in particular positions within written words is an orthographic feature encoded at the letter level, that is statistically abstracted from the bank of lexical entries stored within the orthographic lexicon, any effects of positional letter frequency should be case insensitive (Coltheart, 1978). Our results support this hypothesis.

In addition, the significant correlations found between letter frequency and detection time for letter targets appearing in the exterior positions of the stimulus array must arise from the abstraction of statistical information about positional letter frequency gained from real-word representations stored within the orthographic lexicon, and not from any task-imposed statistics because the frequency with which each of the 25 letters was presented (as either a target or distracter) was equated across the 1,000 experimental trials.

Thus, this study has shown that skilled readers of English encode information about positional letter frequency but frequency information about larger sub-lexical units may also be encoded in a similar manner. This process is likely to emerge during reading acquisition, as the number of lexical entries increases with increased exposure to written words. Accordingly, the sensitivity to positional letter frequency shown by skilled readers may not be observed in early childhood, when children are beginning to learn to read, and may only emerge over the course of reading acquisition as the orthographic lexicon becomes increasingly established. We are currently investigating this possibility.

There appears to be preliminary support for this hypothesis. In reviewing studies of orthographic learning, Castles and Nation (2006) cite two studies that have investigated sensitivity to orthographic regularities in young children in the early stages of formal literacy instruction. Cassar and Treiman (1997) showed sensitivity to orthographic frequency and legality was apparent in 6-year-olds and Kindergarten children. Likewise, Pacton, Perruchet, Fayol and Cleeremans (2001) showed that sensitivity to legality and position of double consonants within words had emerged in French children after just 4 months of formal education. Pacton et al. (2001) argued that children quickly become sensitive to the distributional characteristics of words in written text. Furthermore, they surmised that this sensitivity arises from general learning mechanisms used in other forms of statistical learning (e.g. Bates \& Elman, 1996). Our data suggest that orthographic regularities are encoded within the written word recognition system during the acquisition of reading skill, and modulate the encoding of letter identities and position, even on relatively distal tasks of orthographic processing, such as letter search.

The results of this investigation have a bearing on current theories of the encoding of letter identity and position within written word recognition. Several specific, yet contrasting, models of letter position encoding have been proposed in recent years (see Davis, 2006; Schoonbaert \& Grainger, 2004, for a detailed review). In some respects our findings are most consistent with serial models of letter position encoding (e.g. Whitney, 2001) in which the temporal firing pattern across letter units encodes letter position. However, to be considered comprehensive, models of letter position encoding should take into account the research findings of the current study. This may involve modifying models such that they incorporate: (i) a directional scanning process consistent with reading habits, which for processing English orthography would benefit the encoding of letters in the first position; and (ii) a process of statistical learning at the lexical level, so as to simulate the effects of positional letter frequency information, which for English orthography appear to operate at the initial and final positions of letter strings, as is so 
clearly demonstrated in our study. The suggestive findings of this study, that employed a distal task of orthographic processing, need to be tested in the context of a more direct task of visual word recognition, such as primed lexical decision in which the position and frequency of the prime is systematically manipulated, before strong conclusions in relation to models of written word recognition can be drawn.

\section{Acknowledgements}

The experiment reported in this paper has been published previously in abstract form (Pitchford, Masterson \& Ledgeway, 2004). We would like to thank Jonathan Grainger for discussion of this research. This research was funded in part by a grant to N.J.P from the University of Nottingham.

\section{Notes}

1. Green et al. (1996) manipulated hand of response in English monoscriptals. Participants were slower overall when responding with their left hand (the non-dominant hand for $87.5 \%$ of the participants) and RTs were elevated towards the right of the letter string. As the opposite pattern was not found with participants responding with their right hand we controlled for this asymmetry by fixing hand of response across all participants.

2. Recent research from our laboratory shows that search behaviour for identifying letter targets embedded within random letter strings, pronounceable pseudowords and real words is qualitatively similar (Wagstaffe, 2006).

3. A similar analysis performed across the interior positions only showed limited sensitivity to the most (mean $\mathrm{RT}=688 \mathrm{~ms}$ ) compared to the least (mean $\mathrm{RT}=693 \mathrm{~ms}$ ) frequent letter position, once the acuity advantage at the medial position was removed, $t(24)=-0.689, p=.498$, two-tailed.

\section{References}

Aslin, R. \& Fiser, J. (2004). Feature-learning by infants and adults reveals common constraints on visual feature hierarchies. Perception, 33(Suppl.), 10.

Averbach, E. \& Corriell, A.S. (1961). Short-term memory in vision. Bell Telephone Technical Journal, 40, $19-31$.

Bates, E. \& Elman, J. (1996). Learning rediscovered: A perspective on Saffran, Aslin, and Newport. Science, 274, 1849-1850.

Bouma, H. (1970). Interaction effects in parafoveal letter recognition. Nature, 226, 177-178.

Bouma, H. (1973). Visual interference in the parafoveal recognition of initial and final letters of words. Vision Research, 13, 767-782.

Butler, B.E. (1975). Selective attention and target search with brief visual displays. Quarterly Journal of Experimental Psychology, 27, 467-477.

Butler, B.E. \& Merikle, P.M. (1973). Selective masking and processing strategy. Quarterly Journal of Experimental Psychology, 25, 542-548.

Cassar, M. \& Treiman, R. (1997). The beginnings of orthographic knowledge: Children's knowledge of double letters in words. Journal of Educational Psychology, 89, 631-644.

Castles, A. \& Nation, K. (2006). How does orthographic learning happen? In S. Andrews (Ed.), From inkmarks to ideas: Current issues in lexical processing. Hove: Psychology Press.

Coltheart, M. (1978). Lexical access in simple reading tasks. In G. Underwood (Ed.), Strategies of information processing. London: Academic Press.

Davis, C.J. (1999). The self-organising lexical acquisition and recognition (SOLAR) model of visual word recognition (Doctoral dissertation, University of New South Wales, Sydney, New South Wales, Australia, 1999). Dissertation Abstracts International, 62, 594. 
Davis, C.J. (2006). Orthographic input coding: A review of behavioural data and current models. In S. Andrews (Ed.), From inkmarks to ideas: Current issues in lexical processing. Hove: Psychology Press.

Ehri, L.C. (2005). Learning to read words: Theory, findings and issues. Scientific Studies of Reading, 9, $167-188$.

Estes, W.K. (1972). Interactions of signal and background variables in visual processing. Perception \& Psychophysics, 12, 278-286.

Estes, W.K., Allmeyer, D.H. \& Reder, S.M. (1976). Serial position functions for letter identification at brief and extended exposure durations. Perception \& Psychophysics, 19, 1-15.

Forster, K.I. (1976). Accessing the mental lexicon. In E.C.T. Walker \& R.J. Wales (Eds.), New approaches to language mechanisms. Amsterdam: North Holland.

Grainger, J. \& Jacobs, A.M. (1993). Masked partial-word priming in visual word recognition: Effects of positional letter frequency. Journal of Experimental Psychology: Human Perception and Performance, 19, 951-964.

Grainger, J. \& Jacobs, A.M. (1996). Orthographic processing in visual word recognition: A multiple read-out model. Psychological Review, 103, 518-565.

Grainger, J. \& van Heuven, W.J.B. (2003). Modeling letter position coding in printed word perception. In P. Bonin (Ed.), Mental lexicon: 'Some words to talk about words'. New York: Nova Science Publishers.

Green, D.W., Liow, S.J.R., Tng, S.K. \& Zielinkski, S. (1996). Are visual search procedures adapted to the nature of the script? British Journal of Psychology, 87, 311-326.

Haber, R.N. \& Standing, L. (1969). Location of errors with a poststimulus indicator. Psychonomic Science, 17, 345-346.

Hammond, E.J. \& Green, D.W. (1982). Detecting targets in letters and non-letter arrays. Canadian Journal of Psychology, 36, 67-82.

Humphreys, G.W., Evett, L.J. \& Quinlan, P.T. (1990). Orthographic processing in visual word identification. Cognitive Psychology, 22, 517-560.

Humphreys, G.W., Evett, L.J., Quinlan, P.T. \& Besner, D. (1987). Orthographic priming: Qualitative differences between priming from identified and unidentified primes. In M. Coltheart (Ed.), Attention and performance XII: The psychology of reading. Hillsdale, NJ: Lawrence Erlbaum Associates.

Jones, M.N. \& Mewhort, D.J.K. (2004). Case-sensitive letter and bigram frequency counts from large-scale English corpora. Behavior Research Methods, Instruments, \& Computers, 36, 388-396.

Jordan, T., Thomas, S., Patching, G. \& Scott-Brown, K. (2003a). Assessing the importance of letter pairs in initial, exterior and interior positions in reading. Journal of Experimental Psychology: Learning, Memory and Cognition, 29, 883-893.

Jordan, T., Thomas, S., Patching, G. \& Scott-Brown, K. (2003b). Assessing the importance of letter pairs in reading - parafoveal processing is not the only view: Reply to Inhoff, Radach, Eiter and Skelly (2003). Journal of Experimental Psychology: Learning, Memory and Cognition, 29, 900-903.

Krueger, L.E. (1970). Visual comparison in a redundant display. Cognitive Psychology, 7, 314-357.

Krumhansl, C.L. \& Thomas, E.A.C. (1976). Extracting identity and location information from briefly presented letters arrays. Perception \& Psychophysics, 20, 243-258.

Ktori, M. \& Pitchford, N.J. (in press). Effect of orthographic transparency on letter position encoding: A comparison of Greek and English monoscriptal and biscriptal readers. Language and Cognitive Processes.

Lupker, S.J., Perea, M. \& Davis, C.J. (in press). Transposed-letter effects: Consonants, vowels and letter frequency. Language and Cognitive Processes: Special Issue.

Mason, M. (1975). Reading ability and letter search times: Effects of orthographic structure defined by singleletter positional frequency. Journal of Experimental Psychology: General, 104, 146-166.

Mason, M. (1982). Recognition time for letter and non-letters: Effects of serial position, array size and processing order. Journal of Experimental Psychology: Human Perception and Performance, 8, 724-738.

Mason, M. \& Katz, L. (1976). Visual processing of non-linguistic strings: Redundancy effects and reading ability. Journal of Experimental Psychology: General, 105, 338-348.

Mayzner, M.S. \& Tresselt, M.E. (1965). Tables of single-letter and digram frequency counts for various wordlength and letter-position combinations. Psychonomic Monograph Supplements, 1, 13-30.

McClelland, J.L. \& Johnson, J.L. (1977). The role of familiar units in the perception of words and nonwords. Perception and Psychophysics, 22, 249-261.

McClelland, J.L. \& Rumelhart, D.E. (1981). An interactive activation model of context effects in letter perception: Part 1. An account of basic findings. Psychological Review, 88, 375-407.

McCusker, L.X., Gough, P.B. \& Bias, R.G. (1981). Word recognition inside out and outside in. Journal of Experimental Psychology: Human Perception and Performance, 7, 538-555. 
Merikle, P.M. (1974). Selective backward masking with an unpredictable mask. Journal of Experimental Psychology, 103, 589-591.

Merikle, P.M. \& Coltheart, M. (1972). Selective forward making. Canadian Journal of Psychology, 26, 296302.

Merikle, P.M., Coltheart, M. \& Lowe, D.G. (1971). On the selective effects of a patterned masking stimulus. Canadian Journal of Psychology, 25, 264-279.

Nuerk, H.C., Rey, A., Graf, R. \& Jacobs, A.M. (2000). Phonological sublexical units in visual word recognition. Current Psychology Letters: Behaviour, Brain \& Cognition, 2, 25-36.

Pacton, S., Perruchet, P., Fayol, M. \& Cleeremans, A. (2001). Implicit learning in real world context: The case of orthographic regularities. Journal of Experimental Psychology: General, 130, 401-426.

Perea, M. \& Pollatsek, A. (1998). The effects of neighborhood frequency in reading and lexical decision. Journal of Experimental Psychology: Human Perception and Performance, 24, 767-779.

Peressotti, F. \& Grainger, J. (1995). Letter-position coding in random consonant arrays. Perception \& Psychophysics, 57, 875-890.

Peressotti, F. \& Grainger, J. (1999). The role of letter identity and letter position in orthographic priming. Perception \& Psychophysics, 61, 691-706.

Pitchford, N.J., Masterson, J. \& Ledgeway, T. (2004). Evidence for serial and global encoding of letter identity and position in a visual search task using skilled and dyslexic adult readers. Perception, 33(Suppl.), 150.

Rey, A., Ziegler, J.C. \& Jacobs, A.M. (2000). Graphemes are perceptual reading units. Cognition, 75, B1-B12.

Saffran, J.R. (2001). Words in a sea of sounds: The output of infant statistical learning. Cognition, 81, 149-169.

Saffran, J.R. (2002). Constraints on statistical language learning. Journal of Memory and Language, 47, 172196.

Schoonbaert, S. \& Grainger, J. (2004). Letter position coding in printed word perception: Effects of repeated and transposed letters. Language and Cognitive Processes, 19, 333-367.

Schwantes, F.M. (1978). Stimulus position functions in tachistoscopic identification tasks: Scanning, rehearsal, and order of report. Perception \& Psychophysics, 23, 219-226.

Stevens, M. \& Grainger, J. (2003). Letter visibility and the viewing position effect in visual word recognition. Perception \& Psychophysics, 65, 133-151.

Tydgat, I. \& Grainger, J. (under review). Serial position effects in identification of letters, digits, and symbols: New evidence from the Reicher-Wheeler task. Manuscript submitted for publication.

Van der Heijden, A.H.C. (1992). Selective attention in vision. London: Routledge.

Vellutino, F.R., Scanlon, D.M. \& Tanzman, M.S. (1994). Components of reading ability: Issues and problems in operationalizing word identification, phonological coding and orthographic coding. In G.R. Lyon (Ed.), Frames of reference for the assessment of learning disabilities: New views on measurement issues. Baltimore, MD: Brookes.

Wagstaffe, J.K. (2006). Encoding of letter position in orthographic processing. Unpublished PhD Thesis. University of Nottingham.

Wagstaffe, J.K. \& Pitchford, N.J. (2004). Can crowding and repetition-priming effects account for facilitation of the initial, medial, and terminal letters in visual-search tasks? Perception, 33(Suppl.), 150.

Wagstaffe, J.K., Pitchford, N.J. \& Ledgeway, T. (2005). Does central fixation account for medial letter facilitation in visual search? Perception, 34(Suppl.), 150.

White, M.J. (1976). Order of processing in visual perception. Canadian Journal of Psychology, 30, 140-156.

Whitney, C. (2001). How the brain encodes the order of letters in a printed word: The SERIOL model and selective literature review. Psychonomic Bulletin \& Review, 8, 221-243.

Wolford, G. \& Hollingsworth, S. (1974). Retinal location and string position as important variables in visual information processing. Perception \& Psychophysics, 16, 437-442. 
Appendix A. Letter search functions for each of the 25 letters

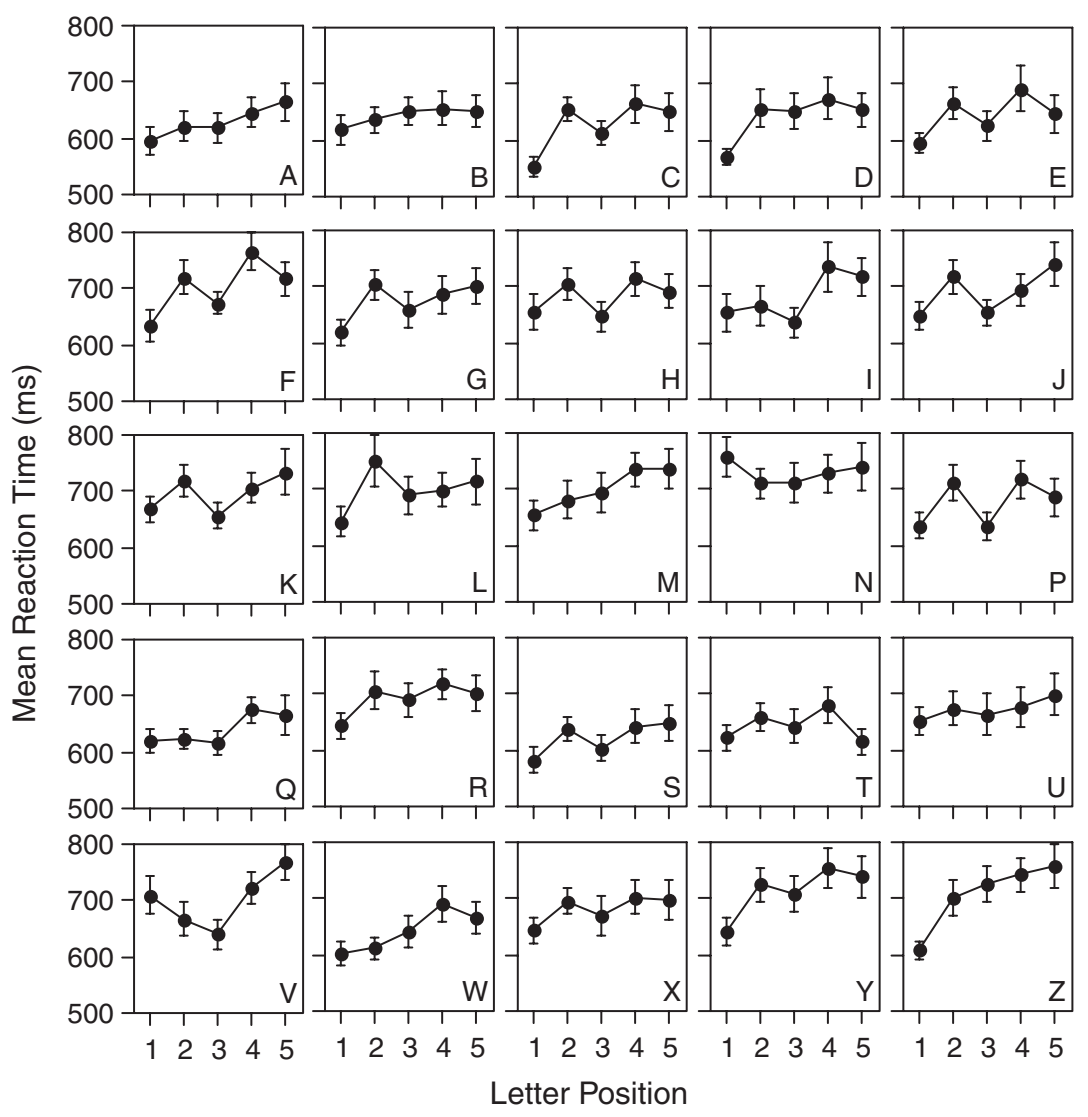

Appendix B. Percentage of the total variance accounted for by each of the four possible components (linear, quadratic, cubic, quartic) for each of the 25 letters

\begin{tabular}{lcccc}
\hline Letter & \multicolumn{4}{c}{ Percentage of total variance } \\
\cline { 2 - 5 } & Linear & Quadratic & Cubic & Quartic \\
\hline A & $\mathbf{9 3}$ & 0 & 2 & 5 \\
B & $\mathbf{7 6}$ & 23 & 1 & 0 \\
C & $\mathbf{4 9}$ & 16 & 7 & 28 \\
D & $\mathbf{5 2}$ & 37 & 3 & 8 \\
E & 30 & 21 & 0 & 49 \\
F & $\mathbf{4 5}$ & 13 & 0 & 42 \\
G & $\mathbf{4 2}$ & 7 & 28 & 23 \\
H & 19 & 1 & 1 & $\mathbf{9 9}$ \\
I & $\mathbf{5 4}$ & 5 & 8 & 25 \\
J & $\mathbf{3 9}$ & 4 & 19 & $\mathbf{4 2}$ \\
K & 32 & 15 & $\mathbf{5 0}$ & 22 \\
L & 13 & 1 & 1 & 3 \\
M & $\mathbf{9 5}$ & $\mathbf{8 1}$ & 17 & 0 \\
N & 2 & & &
\end{tabular}


Appendix B. Continued

\begin{tabular}{lcccc}
\hline Letter & \multicolumn{3}{c}{ Percentage of total variance } \\
\cline { 2 - 5 } & Linear & Quadratic & Cubic & Quartic \\
\hline P & 18 & 34 & 2 & $\mathbf{7 6}$ \\
Q & $\mathbf{6 6}$ & 4 & 11 & 19 \\
R & $\mathbf{4 8}$ & 27 & 4 & 21 \\
S & $\mathbf{5 7}$ & 2 & 10 & 31 \\
T & 0 & $\mathbf{5 6}$ & 10 & 34 \\
U & $\mathbf{7 7}$ & 3 & 15 & 5 \\
V & 30 & $\mathbf{5 9}$ & 2 & 9 \\
W & $\mathbf{7 9}$ & 3 & 16 & 2 \\
X & $\mathbf{5 4}$ & 17 & 6 & 32 \\
Y & $\mathbf{6 5}$ & 13 & 2 & 16 \\
Z & $\mathbf{8 3}$ & 3 & 1 \\
\hline
\end{tabular}

For each letter the trend that captures the most variance is shown in bold.

Received 12 March 2007; revised version received 11 September 2007.

Address for correspondence: Dr Nikki Pitchford, School of Psychology, University of Nottingham, University Park, Nottingham NG7 2RD, UK. E-mail: nicola.pitchford@ nottingham.ac.uk 